07,13

\title{
Влияние поверхностных эффектов на изгиб и колебания нанопленок
}

\author{
(C) М.А. Ильгамов \\ ${ }^{1}$ Институт машиноведения им. А.А. Благонравова РАН, \\ Москва, Россия \\ ${ }^{2}$ Башкирский государственный университет, \\ Уфа, Россия \\ ${ }^{3}$ Институт механики Уфимского федерального исследовательского центра РАН, \\ Уфра, Россия \\ E-mail: ilgamov@anrb.ru
}

Поступила в Редакцию 19 фревраля 2019 г.

В окончательной редакции 1 апреля 2019 г.

Принята к публикации 2 апреля 2019 г.

\begin{abstract}
Статический цилиндрический изгиб нанопленок рассмотрен в линейной и нелинейной постановках. Определены спектр частот изгибных колебаний и параметрический резонанс. При этом учитываются два поверхностных эффекта. Первый из них связан с различающимися упругими свойствами в приповерхностном слое и в основном объеме материала. Он проявляется при растяжении и изгибе пленок нанометровых толщин. Второй эффект обусловлен появляющейся при изгибе разностью площадей выпуклой и вогнутой поверхностей, на которые действуют давления газов. Этот эффект проявляется тем сильнее, чем больше отношение среднего давления к модулю упругости материала и длины пленки к ее толщине. Имеют значение также условия нагружения торцевых поверхностей пленки, а также деформация по толщине пленки под действием среднего давления. Положительное среднее избыточное давление (сжатие) приводит к увеличению эффективной жесткости, уменьшению прогиба, возрастанию собственных частот. Отрицательное среднее давление (вакуумирование) уменьшает жесткость и собственные частоты. Показано, что в этом случае может иметь место изгиб пленки в результате потери продольной устойчивости. Колебания среднего давления приводят к параметрическому усилению изгибных колебаний. Эти результаты не могут быть получены на основе классических уравнений изгиба тонких пластин и пленок.
\end{abstract}

Ключевые слова: поверхностные эффекты, изгиб, устойчивость, спектр частот, параметрический резонанс.

DOI: $10.21883 /$ FTT.2019.10.48255.381

\section{1. Введение}

Нанопленки и нанопроволоки находят применение в электронных, оптоэлектронных и электромеханических устройствах, в литографии, в качестве химических и биологических сенсоров и т. д. [1-4]. Изучение их эксплуатационных свойств, в частности, статической и динамической деформации пленки под действием приложенных сил, спектра частот, устойчивости формы представляет большой интерес. Как показано экспериментально и теоретически в работах $[5,6]$, а также в последующих исследованиях, при толщинах пленки порядка $10 \mu \mathrm{m}$ и меньше начинает влиять на деформацию поверхностный эффект, связанный с различием упругих характеристик в тонком слое около поверхности и в основном объеме тела. Укажем на следующие исследования [7-11] в этом направлении, в которых приводится также обзор литературы. В частности, статический изгиб пленки, устойчивость ее формы, свободные колебания рассматриваются в [10-13].

Согласно этим работам продольная сила $N$, изгибающий момент $M$ при цилиндрическом изгибе пленки в направлении оси $x$ выражаются через деформацию $\varepsilon$ срединной поверхности и ее кривизну $\kappa$ формулами

$$
\begin{gathered}
N=K_{*} \varepsilon, \quad M=D_{*} \kappa, \quad K_{*}=K(1+\beta), \\
D_{*}=D(1+3 \beta), \quad K=\frac{E h}{1-v^{2}}, \\
D=\frac{E h^{3}}{12\left(1-v^{2}\right)}, \quad \beta=\frac{2\left(\lambda_{0}+2 \mu_{0}\right)\left(1-v^{2}\right)}{E h},
\end{gathered}
$$

где $E, v-$ модуль упругости и коэффициент Пуассона для основного объема пленки, определяемые в классической теории упругости [14-17], $\lambda_{0}, \mu_{0}$ - поверхностные константы Ламе, $h$ - толщина, $K_{*}, D_{*}$ - эффективные жесткости при растяжении и изгибе. Безразмерный параметр $\beta$ здесь записан без малых членов, которые присутствуют в работах [7-11]. Отметим, размерность модуля $E$ в $\mathrm{MPa}$, а констант $\lambda_{0}$ и $\mu_{0}-$ в $\mathrm{MPa} \cdot \mathrm{m}$. Толщина приповерхностного слоя не рассматривается. Для двух материалов в $[7,10]$ приведены значения $h \beta=765 \mathrm{~nm}$ (железо) и $h \beta=-4.5 \mathrm{~nm}$ (железо на стеклянном субстрате). Таким образом, эффективные жесткости $K_{*}$ и $D_{*}$ могут быть как больше, так и меньше значений жесткостей $K$ и $D$. Как видно из (1), эффективная 




Рис. 1. Собственные формы и частоты изгибных колебаний консольного резонатора. Пленка состоит из двух слоев толщинами $0.3 \mu \mathrm{m}(\mathrm{Au})$ и $1.5 \mu \mathrm{m}\left(\mathrm{SiN}_{x}\right)[1,2]$.

изгибная жесткость для первого приведенного значения $h \beta$ начинает заметно увеличиваться при толщине $h<10^{4} \mathrm{~nm}$, а для второго значения $h \beta-$ уменьшается при $h<10^{2} \mathrm{~nm}$.

Второй поверхностный эффект связан с образующейся разностью площадей верхней и нижней поверхностей горизонтально расположенной пленки в результате ее изгиба. Эта разность площадей приводит к появлению дополнительной распределенной поперечной силы. Поперечная сила зависит также от давлений $p_{1}$ и $p_{2}$, действующих на нижнюю и верхнюю поверхности. Начиная с трудов Дж. Рэлея [14] и последующих исследований вплоть до современной литературы (например, [15-18]) принимается, что поперечная распределенная сила $q$ на тонкую пластину равна $q=g \rho h+p_{2}-p_{1}$, где $g-$ гравитационное ускорение, $\rho-$ плотность. Если учитывать указанную выше разность площадей выпуклой и вогнутой поверхностей пленки, то общая распределенная поперечная сила $q$ в случае цилиндрического изгиба равна [19-21]

$$
q=g \rho h+p_{2}-p_{1}+p_{m} h \kappa, \quad p_{m}=\left(p_{1}+p_{2}\right) / 2 .
$$

Настоящая работа посвящена одновременному учету указанных выше поверхностных эффектов на статический изгиб и изгибные колебания нанопленок, находящихся под давлением газов и не являющихся покрытием объемных материалов [22-24]. При этом учитывается их сопротивление изгибающему моменту, как это делается в [7-11]. Этот учет необходим ввиду изменения отношения длины волны к толщине пленок в широких пределах. Например, в экспериментах, описанных в [1,2], отношение длины консольного резонатора к толщине изменяется от 35 до 320 (рис. 1).

\section{2. Постановка задачи}

Пленка, находящаяся в горизонтальном положении, закреплена по кромкам $x=0, L$ и имеет большую протяженность в направлении оси у по сравнению с пролетом $L$ (рис. 2). Условия закрепления относительно компонент перемещения $u(x, t), w(x, t)$ принимаем в виде

$$
u=0, \quad w=\partial^{2} w / \partial x^{2}=0 \quad(x=0, L) .
$$

Таким образом, будем рассматривать цилиндрический изгиб пленки под действием ее собственного веса $g \rho h$, давлений газов $p_{0}+p_{1}$ и $p_{0}+p_{2}$ на нижнюю и верхнюю поверхности. По поверхностям пленки они не изменяются. Для определенности под $p_{0}$ будем подразумевать атмосферное давление, которое действует также на поверхности кромок $x=0, L$ пленки. Это состояние с компонентами перемещения $u_{0}, w_{0}$ под всесторонним давлением считаем ненапряженным.

Избыточные давления $p_{1}$ и $p_{2}$ на кромки пластины не действуют. Могут быть устройства опор, где действие их на площади кромок не передается в область $0 \leq x \leq L$ (рис. 2). Положительное направление оси $z$, поперечной силы $q$ и перемещения $w$ принято вниз.

Для простоты иллюстрации указанных эффектов не учитываем излучение энергии пленкой в окружающую среду. Это же относится и к присоединенной массе среды, вовлекаемой в движение на контактных поверхностях пленки. Эти допущения могут быть приемлемы в случае легких газов, вакуумирования поверхностей и т.д. В статических задачах изгиба эти ограничения снимаются. Движение газов вдоль пленки может оказывать влияние на ее деформацию даже в указанных выше условиях $[16,17]$. Здесь принимается, что такого движения нет. При этих допущениях распределенная поперечная сила на пленку определяется выражением (2).

Уравнение изгиба пленки имеет вид [15]

$$
\frac{\partial^{2} M}{\partial x^{2}}-N \frac{\partial^{2}\left(w_{0}+w\right)}{\partial x^{2}}+\rho h \frac{\partial^{2} w}{\partial t^{2}}=q
$$

Здесь под $M$ и $N$ будем подразумевать эффективные изгибающий момент и продольную силу по (1). Таким образом, коэффициентами $D_{*}, K_{*}$ учитывается первый поверхностный эффект, а в составе распределенной поперечной силы $q$ содержится второй поверхностный эффект.

Деформация растяжения пленки происходит как за счет отклонения от плоскости, так и сжатия по толщине под действием среднего давления $p_{m}$. Отметим, в (1)

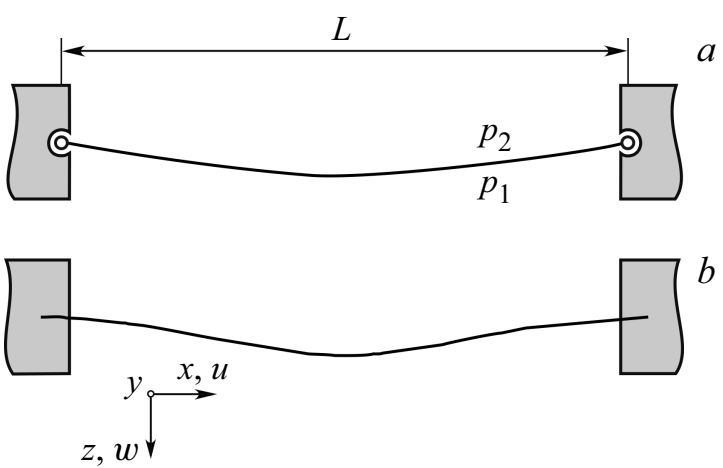

Pис. 2. Примеры закреплений пленки: $a-$ действие давления на площади кромок не создает продольную силу в пленке; $b-$ кромки изолированы от внешней среды и на них не действует давление. 
выражение для силы $N$ получено в предположении отсутствия деформации по толщине $\left(\varepsilon_{z}=0\right)$, принимаемом в теории тонких пластин и пленок [14-18]. Ввиду того, что в рассматриваемой модели допускаются немалые значения среднего давления $p_{m}$, необходимо учитывать влияние поперечной деформации $\varepsilon_{z}$ на силу $N$. В приближенном анализе закон Гука представим с применением эффективной жесткости $K_{*}$

$$
N=K_{*}\left(\varepsilon+v \varepsilon_{z}\right), \quad N_{z}=K_{*}\left(\varepsilon_{z}+v \varepsilon\right) .
$$

Учитывая выражение для $K_{*}$ по (1) и $N_{z}=-p_{m} h$, находим из (5)

$$
N=E h \varepsilon(1+\beta)-v p_{m} h .
$$

Подставив в (6) выражение продольной деформации $[15-18] \varepsilon=\partial u / \partial x+(\partial w / \partial x)^{2} / 2$, проинтегрировав по $x$ от нуля до $L$ и удовлетворив условиям (3) относительно $u(x)$, получаем

$$
N=\frac{E h(1+\beta)}{2 L} \int_{0}^{L}\left(\frac{\partial w}{\partial x}\right)^{2} d x-v p_{m} h .
$$

Подстановка в (4) выражений для $M, N, q$ в соответствии с (1), (2), (7) и зависимости $\kappa=\partial^{2} w / \partial^{2} x$ приводит к уравнению

$$
\begin{gathered}
D(1+3 \beta) \frac{\partial^{4} w}{\partial x^{4}}-\left((1-v) p_{m} h+\frac{E h(1+\beta)}{2 L} \int_{0}^{L}\left(\frac{\partial w}{\partial x}\right)^{2} d x\right) \\
\times \frac{\partial^{2}\left(w_{0}+w\right)}{\partial x^{2}}+\rho h \frac{\partial^{2} w}{\partial t^{2}}=p_{e} \\
p_{e}=g \rho h+p_{2}-p_{1} .
\end{gathered}
$$

Если одна из опор допускает свободное скольжение вдоль плоскости пленки, то вместо условия $u(0, L)=0$ в (3) нужно положить равной нулю продольную силу $N$. Тогда в рамках рассматриваемой модели уравнение (4) не будет содержать второй член, так как будет $N=0$ по всей длине. При этом исчезает и влияние начального отклонения $w_{0}$ на изгиб при приложении давлений $p_{1}$ и $p_{2}$. В уравнении (8) нужно положить равными нулю $w_{0}$, $v p_{m} h$ и член, содержащий интеграл.

В случае действия на площадь одной из кромок давления $p_{m}$ возникает дополнительная продольная сила сжатия $N=-p_{m} h$. При этом в (8) нужно исключить член $-v p_{m} h$. Тогда в уравнении $(8)$ отсутствует весь второй член. На изгиб пленки в этом случае влияет только первый поверхностный эффект.

\section{3. Статический изгиб}

Влияние указанных поверхностных эффектов наиболее наглядно может быть показано для статического

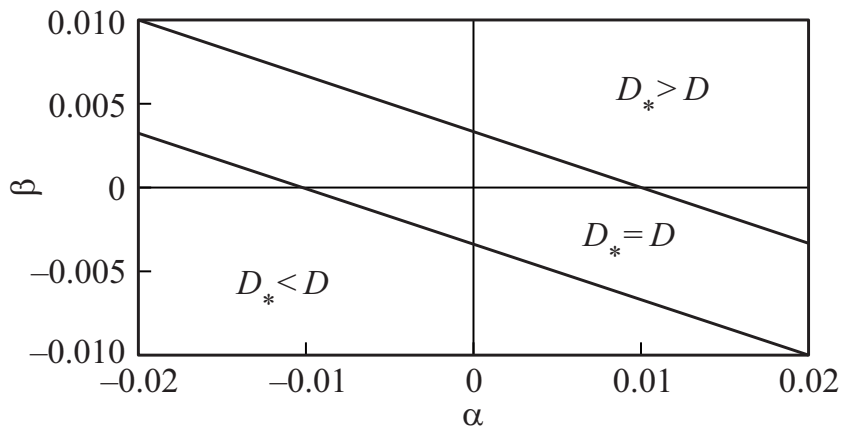

Рис. 3. Области безразмерных параметров поверхностных эффектов, в которых эффективная жесткость $D_{*}$ больше, равна и меньше, чем изгибная жесткость $D(1)$, вводимая в теории упругости [15].

изгиба, при котором функции $w_{0}$ и $w$, удовлетворяющие условиям (3), могут быть приняты

$$
w_{0}=W_{0} \sin (\pi x / L), \quad w=W \sin (\pi x / L)
$$

Подставив (9) в (8), умножив это уравнение на $\sin (\pi x / L)$ и проинтегрировав в пределах от 0 до $L$, получаем

$$
\begin{array}{r}
(1+\beta) \gamma W^{3}+(1+\alpha+3 \beta) W=\mu-\alpha W_{0}, \\
\alpha=\frac{(1-v) p_{m} h L^{2}}{\pi^{2} D}, \quad \gamma=\frac{E h}{D}, \quad \mu=\frac{4 p_{e} L^{4}}{\pi^{5} D} .
\end{array}
$$

Сначала рассмотрим малые значения стрелы прогиба $W$. Опустив в (10) нелинейный член, имеем

$$
W=\frac{\mu-\alpha W_{0}}{1+\alpha+3 \beta} .
$$

При постоянном значении $\mu$ и $\alpha>0, \beta>0$ дополнительный прогиб $W$ меньше, чем классическое решение $W=\mu$, а при $\alpha<0, \beta<0-$ больше. Могут быть случаи, когда $\alpha$ и $\beta$ разных знаков. Тогда эффективная жесткость $D(1+\alpha+3 \beta)$ возрастает и $W$ уменьшается при $\alpha+3 \beta>0$. Если принять необходимым учет поверхностных эффектов, начиная со значения $\alpha+3 \beta>10^{-2}$, то получаем три области, в которых эффективная изгибная жесткость $D_{*}$ по разному отличается от обычной жесткости $D$ (рис. 3 ).

Как следует из (1) и (10), безразмерный параметр $\beta$ обратно пропорционален толщине $h$ пленки, а параметр $\alpha-$ квадрату отношения $L / h$. Знак параметра $\beta$ зависит о материала, а параметра $\alpha-$ от знака среднего давления $p_{m}$ (при сжатии по толщине пленки принимается $p_{m}>0$, при вакуумировании ее поверхностей $\left.p_{m}<0\right)$. Дополнительный прогиб (11) при отсутствии перепада давления $\left(p_{e}=0, \mu=0\right)$ имеет отрицательное значение, что означает выпрямление изогнутой пленки. При этом общее отклонение от плоскости под действием среднего давления составляет $W_{0}+W=W_{0}\left[(1+3 \beta)(1+3 \beta+\alpha)^{-1}\right]$. 
Рассмотрим два варианта численных данных $[7,10]$ : $\beta=765 / h, E=5.62 \cdot 10^{4} \mathrm{MPa}, v=0.25$ и $\beta=-4.5 / h$, $E=17.73 \cdot 10^{4} \mathrm{MPa}, v=0.27$. Пусть $L / h=50, W_{0}=0$. При $p_{m}=1 \mathrm{MPa}$ в обоих вариантах данных параметр $\alpha$ мал по сравнению с единицей. Если при этом $h<10^{4} \mathrm{~nm}$, то в первом варианте поверхностный эффект, связанный с материалом пленки, приводит к увеличению эффективной жесткости, во втором варианте он не проявляется. При $p_{m}=20 \mathrm{MPa}$ параметр $\alpha$ для рассматриваемых материалов равен $\alpha \approx 0.89$ и $\alpha \approx 0.28$. Для этих значений $\alpha$ и первого материала толщиной $h=5 \cdot 10^{3} \mathrm{~nm}$ из (11) имеем $W=0.43 \mu$ и $W=0.74 \mu$ (вместо $W=\mu$, что получается без учета поверхностных эффектов).

При $\alpha+3 \beta \geq-1$ и $\mu \neq \alpha W_{0}$ линейное решение (11) неограниченно возрастает. Значение $\alpha_{*}=-(1+3 \beta)$ можно считать критическим. Критическое среднее избыточное давление с учетом (10) можно представить в виде

$$
p_{m}^{*}=-\frac{\pi^{2} D(1+3 \beta)}{h L^{2}(1-v)} \approx-\frac{E(1+3 \beta)}{1-v}\left(\frac{h}{L}\right)^{2} .
$$

Вопрос состоит в том, является ли значение $p_{m}^{*}$ по (12) реальным для действительных входных данных. Оно может быть выражено также в виде $h p_{m}^{*}=-P_{E}(1+3 \beta) /(1-v)$, где $P_{E}=\pi^{2} D / L^{2}$ эйлерово значение критической продольной сжимающей силы.

При принятых выше данных для обоих материалов значение $p_{m}^{*}$ по абсолютной величине намного превосходит возможное отрицательное избыточное давление в газах $-0.1 \mathrm{MPa}$. Но для $L / h \geq 2 \cdot 10^{3}$ и первого материала $p_{m}^{*} \leq-0.062 \mathrm{MPa}$. Таким образом, вакуумирование поверхностей пленки может приводить к ее выпучиванию по синусоиде в случае больших отношений $L / h$ и относительно малого модуля упругости материала $E$.

Рассмотрим случай $\mu=0, W_{0}=0$, когда пленка не изгибается. Однако при $1+\alpha+3 \beta<0$ происходит закритический изгиб. Прогиб определяется из (10) $W= \pm \sqrt{-(1+\alpha+3 \beta) /(\gamma(1+\beta))}$. Имеется равная возможность отклоняться вниз и вверх.

Когда $W \geq h$ и $\alpha \sim 1, \beta \sim 1, \mu \neq \alpha W_{0}$, преобладающим становится первый член в уравнении (10). При этом

$$
W \approx\left[\frac{\mu-\alpha W_{0}}{\gamma(1+\beta)}\right]^{1 / 3} .
$$

Как указывалось выше, в случае опорного устройства, допускающего свободное скольжение, продольная сила $N$ отсутствует. Уравнение $(8)$ становится линейным, поэтому решение (13) не имеет места. Кроме того, параметр $\alpha$ по (10) не содержит коэффициент Пуассона $v$, критическое значение $p_{m}^{*}(12)$ уменьшается. В (11) исключается член $\alpha W_{0}$. В том случае, когда на площади кромок действует давление $p_{m}$, то исчезает влияние параметра $\alpha$ и согласно (11) стрела погиба уменьшается: $W=\mu /(1+3 \beta)$.

\section{4. Изгибные колебания}

Рассмотрим сначала собственные колебания пленки, закрепленной так, что удовлетворяются условия (3). Опустив нелинейный член, а также члены $p_{e}$ и $W_{0}$ в уравнении (8) и подставив в него функцию прогиба

$$
w=\sin (n \pi x / L) \sin \omega_{n} t, \quad n=1,2, \ldots, .
$$

находим собственные частоты

$$
\omega_{n}=\Omega_{n} \sqrt{1+\frac{\alpha}{n^{2}}+3 \beta}, \quad \Omega_{n}=\left(\frac{n \pi}{L}\right)^{2} \sqrt{\frac{D}{\rho h}} .
$$

Здесь $\Omega_{n}-$ собственные частоты изгибных колебаний пленки без поверхностных эффектов. Формула (15) без параметра $\alpha$ получена в [7]. Как видно из (15), эффект, обусловленный средним давлением, быстро убывает для высших гармоник. Положительные безразмерные параметры $\alpha, \beta$ повышают собственные частоты, отрицательные - понижают. Это происходит ввиду соответствующего изменения эффективной изгибной жесткости. При $n=1, \alpha=-1-3 \beta$ собственная частота $\omega_{1}$ обращается в нуль, что имеет место при достижении средним давлением критического значения (12).

Определение численных значений параметра $\beta$ для пленок из разных материалов представляет очевидные сложности. Одним из способов его определения может быть экспериментальное значение собственной частоты $\left(\omega_{1}\right)$ в отсутствии среднего избыточного давления на пленку $(\alpha=0)$. Тогда $3 \beta=\left(\left(\omega_{1}\right) / \Omega_{1}\right)^{2}-1$. При этом предполагаем, что известны входные параметры для вычисления частоты $\Omega_{1}$.

Отметим, в отличие от параметра $\beta$ значение параметра $\alpha$ допускает управление. В частности, при изменении среднего давления во времени могут быть возбуждены изгибные колебания пленки. Случай приложения скачкообразно повышающегося давления на обе поверхности пластины $\left(p_{1}=p_{2}\right)$, а также скачкообразно снижающегося давления рассмотрен в [21]. При этом не учитывался поверхностный эффект, связанный с материалом $(\beta=0)$. Если происходят колебания давлений $p_{1}-P_{1} \cos \omega t, p_{2}-P_{2} \cos (\omega t+\varphi)$, то могут возбуждаться совместные вынужденные и параметрические колебания пленки.

Рассмотрим случай $w_{0}=0, g=0, p_{1}=p_{2}, P_{1}=P_{2}$, $\varphi=0$. При этом $p_{m}=p_{1}, p_{e}=0(\mu=0)$, среднее избыточное давление $p_{m}-P \cos \omega t$ (в рамках классических моделей пластин и пленок в этом случае есть только нулевое решение). Соответственно в уравнении (8) вместо параметра $\alpha$ поставим $\alpha-\alpha p \cos t$. Решение уравнения (8) по $x$ представим в виде первой гармоники (9), где теперь амплитуда $W(t)$ является функцией времени. Вместо (10) уравнение (8) приводится к виду

$\frac{1}{\Omega_{1}^{2}} \frac{d^{2} W}{d t^{2}}+\left(1+\alpha+3 \beta-\alpha_{p} \cos \omega t\right) W+(1+\beta) \gamma W^{3}=0$, 


$$
\alpha_{p}=\frac{(1-v) P h L^{2}}{\pi^{2} D},
$$

где $\Omega_{1}$ определяется по (15) при $n=1$. Колебания, происходящие при главном параметрическом резонансе $\omega \approx 2 \Omega_{1} \sqrt{1+\alpha+3 \beta}$, представим приближенно в виде [25]

$$
W=a \sin \frac{\omega t}{2}+b \cos \frac{\omega t}{2}
$$

Подставив (17) в (16) без последнего члена и сохранив в нем только члены с функциями от аргумента $\omega t / 2$, находим область параметрического резонанса

$$
\frac{\omega}{2 \Omega_{1}}=\sqrt{1+\alpha+3 \beta \pm \frac{\alpha_{p}}{2}} .
$$

Как видно из (18), поверхностные эффекты в зависимости от знаков $\alpha+3 \beta$ сдвигают область резонанса в большую или меньшую сторону, не оказывая влияния на ширину области. С уменьшением переменной части среднего избыточного давления $P \cos \omega t$ эта область сужается. При отсутствии этой части область исчезает, параметрические колебания не возникают. Это видно и по уравнению (16).

C учетом последнего члена уравнения (16) и функции (17) находим амплитуду установившихся колебаний

$$
\begin{aligned}
A= & \sqrt{a^{2}+b^{2}}=\frac{2}{\sqrt{3 \gamma(1+\beta)}} \\
& \times \sqrt{\left(\frac{\omega}{2 \Omega_{1}}\right)^{2}-\left(1 \pm \frac{\alpha_{p}}{2}\right)(1+\alpha+3 \beta)^{2}} .
\end{aligned}
$$

Решение с членом $+\alpha_{p} / 2$ неустойчиво. В (19) необходимо оставить член, содержащий $-\alpha_{p} / 2$. Отнесенная к толщине пленки амплитуда с учетом обозначений (10) может быть выражена также через частоту $\omega_{1}$

$$
\frac{A}{h}=\frac{1+\alpha+3 \beta}{3 \sqrt{\left(1-v^{2}\right)(1+\beta)}} \sqrt{\left(\frac{\omega}{2 \omega_{1}}\right)^{2}-1+\frac{\alpha_{p}}{2}} .
$$

Отсюда видно возрастание относительной амплитуды параметрических колебаний с увеличением параметров поверхностных эффектов, однако нет сдвига относительно $\omega /\left(2 \omega_{1}\right)$, так как они учтены в выражении для частоты $\omega_{1}$.

\section{5. Заключение}

Поверхностный эффект, связанный с различием упругих свойств в приповерхностном слое и в основном объеме материала, приводит к изменению жесткостей при растяжении и изгибе пленки. Этот эффект возрастает обратно пропорционально толщине пленки $h$. Его влияние начинается с малых микроразмеров и наноразмеров $h$, может как повышать жесткость, так и понижать. В уравнении изгиба пленки (8) эффект учитывается безразмерным параметром $\beta$, приведенным в (1). Он не зависит от граничных условий. Необходимо отметить отсутствие в литературе числовых данных для многих материалов.

Второй рассматриваемый эффект обусловлен взаимодействием разности площадей выпуклой и вогнутой поверхностей пленки при изгибе и среднего давления $p_{m}=\left(p_{1}+p_{2}\right) / 2$. Здесь $p_{1}, p_{2}-$ избыточные давления, действующие на поверхности. Значения $p_{1}, p_{2}, p_{m}$ могут быть положительными (сжатие пленки по толщине) и отрицательными (вакуумирование поверхностей). В уравнении (8) эффект учитывается безразмерным параметром $\alpha$, приведенным в (10). Его значение тем больше, чем больше отношение $p_{m}$ к модулю упругости материала $E$ и квадрат отношения длины пленки $L$ к ее толщине $h$.

На эффективную жесткость при растяжении $K_{*}$ пленки влияет только первый эффект, а на эффективную изгибную жидкость $D_{*}$ - оба фактора $\left(K_{*}=K(1+\beta)\right.$, $D_{*}=D(1+\alpha+3 \beta)$, где $K$ и $D-$ жесткости, вводимые в классической теории упругости [15]).

В отличие от первого эффекта влияние на изгиб второго может быть значительным не только для микрои нанопленок. Это объясняется тем, что второй эффект зависит от отношения $(L / h)^{2}$. Кроме того он существенно зависит от условий закрепления пленки по краям. Например, при условиях защемления краев пленки параметр $\alpha$ меньше в четыре раза по сравнению со значением (10) при условиях свободного опирания. Объяснение этого результата состоит в том, что при условиях свободного опирания кривизна поверхности имеет один знак, а при защемлении происходит смена знаков кривизны по поверхности.

При положительных значениях параметров $\alpha$ и $\beta$ статический изгиб пленки меньше, а собственные частоты выше, чем при $\alpha=0, \beta=0$. Наоборот, при $\alpha<0, \beta<0$ статический прогиб возрастает, а собственная частота уменьшается. При вакуумировании поверхностей пленки может быть достигнуто критическое значение параметра $\alpha$ и соответствующее значение среднего давления $p_{m}$, когда происходит выпучивание пленки, как при осевом сжатии. При этом первая собственная частота колебаний снижается до нуля. Зависимость собственных частот от второго поверхностного эффекта быстро уменьшается для высших гармоник.

При изменяющихся во времени значениях давлений на поверхности пленки возбуждаются ее вынужденные и параметрические колебания. Последние имеют место даже при отсутствии перепада давлений. Параметрический резонанс возникает при колебаниях среднего избыточного давления. Такие результаты не могут быть получены без учета поверхностного эффекта, обусловленного взаимодействием среднего давления и разности площадей поверхностей пленки, появляющейся при изгибе.

Приведенные результаты показывают, что при анализе деформации нанопленок и их взаимодействия с окружающей газовой средой должны учитываться рассмотренные в статье поверхностные эффекты. 


\section{Финансирование работы}

Работа выполнена в рамках государственного задания (проекты № 0246-2019-0088, № 0049-2015-0040) и РФФИ (грант № 18-01-00150).

\section{Список литературы}

[1] A. Raman, J. Melcher, R. Tung. Nano Today. 3, 1-2, 20 (2008).

[2] K. Eom, H.S. Park, D.S. Yoon, K. Kwon. Phys. Rep. 503, 4-5, 115 (2011).

[3] R. Elnathan, M. Kwiat, F. Patolsky, N.H. Voelcker. Nano Today 9, 2, 172 (2014).

[4] L.J. Guo. Adv. Mater. 19, 4, 495 (2007).

[5] P.E. Sheehan, C.M. Lieber. Science 272, 1156 (1996).

[6] E. Wong, P.E. Sheehan, C.M. Lieber. Science 277, 1971 (1997).

[7] M.E. Gurtin, A.I. Murdoch. Int. J. Solids Struct. 14, 431 (1978).

[8] R.E. Miller, V.B. Shenoy. Nanotechnology 11, 139 (2000).

[9] C.T. Sun, H. Zhang. J. Appl. Phys. 93, 1212 (2003).

[10] P. Sharma, S. Ganti, N. Bhate. Appl. Phys. Lett. 82, 535 (2003).

[11] L.H. He, C.W. Lim, B.S. Wu. Int. J. Solids Struct. 41, 847 (2004).

[12] C.W. Lim, L.H. He. Int. J. Mech. Sci. 46, 11, 1715 (2004).

[13] Y. Huang, X.F. Li. Int. J. Struct. Stab. Dyn. 12, 4, 120027 (2012).

[14] J.W. Rayleigh. The Theory of Sound. Macmillan and Company,London (1894). V. I. 500 p.

[15] Л.Д. Ландау, Е.М. Лифшиц. Теория упругости. Наука. М. (1987). $248 \mathrm{c}$

[16] E.H. Dowell. Aeroelasticity of Plates and Shells. NIP, Leyden (1975). $139 \mathrm{p}$

[17] E.H. Dowell, M.A. Ilgamov. Studies in Nonlinear Aeroelasticity. SV, N.Y., London, Tokyo (1988). 456 p.

[18] H.Sh. Shen. Postbuckling Behavior of Plates and Shells. Jiao Tong University, Shanghai (2017). 675 p.

[19] М.А. Ильгамов. ДАН. 476, 4, 402 (2017).

[20] М.А. Ильгамов. ДАН. 480, 5, 542 (2018).

[21] М.А. Ильгамов. Акустический журн. 64, 5, 598 (2018).

[22] Г.А. Малыгин. ФТТ 52, 48 (2010).

[23] Г.А. Малыгин. ФТТ 54, 523 (2012).

[24] Г.А. Малыгин. ФТТ 57, 1507 (2012).

[25] Л.Д. Ландау, Е.М. Лифшиц. Механика. Наука, М. (1988). 216 c.

Редактор Т.Н. Василевская 